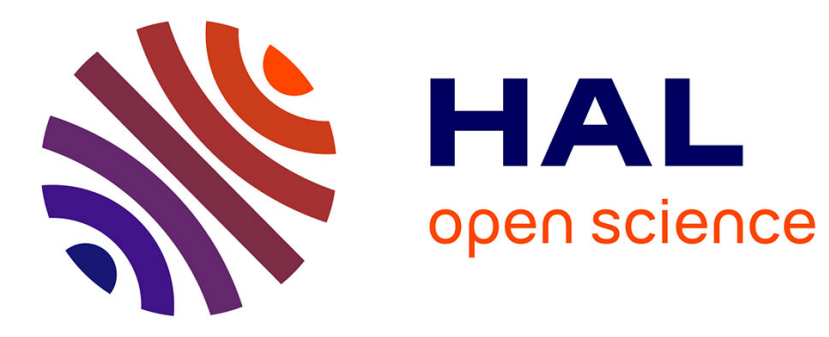

\title{
Multiple time-scales of adaptation in the auditory system as revealed by human evoked potentials
}

Jordi Costa-Faidella, Sabine Grimm, Lavinia Slabu, Francisco Díaz-Santaella, Carles Escera

\section{- To cite this version:}

Jordi Costa-Faidella, Sabine Grimm, Lavinia Slabu, Francisco Díaz-Santaella, Carles Escera. Multiple time-scales of adaptation in the auditory system as revealed by human evoked potentials. Psychophysiology, 2010. hal-01898520

\section{HAL Id: hal-01898520 \\ https://hal.science/hal-01898520}

Submitted on 18 Oct 2018

HAL is a multi-disciplinary open access archive for the deposit and dissemination of scientific research documents, whether they are published or not. The documents may come from teaching and research institutions in France or abroad, or from public or private research centers.
L'archive ouverte pluridisciplinaire HAL, est destinée au dépôt et à la diffusion de documents scientifiques de niveau recherche, publiés ou non, émanant des établissements d'enseignement et de recherche français ou étrangers, des laboratoires publics ou privés. 


\title{
Multiple time-scales of adaptation in the auditory system as revealed by human evoked potentials
}

\author{
Jordi Costa-Faidella, Sabine Grimm, Lavinia Slabu, Francisco Díaz-Santaella \& \\ Carles Escera
}

\begin{abstract}
Author affiliation: Institute for Brain, Cognition and Behavior (IR3C) and Cognitive Neuroscience Research Group, Department of Psychiatry and Clinical Psychobiology, University of Barcelona, Catalonia-Spain

Corresponding author: Carles Escera, PhD, Professor, Cognitive Neuroscience Research Group, Department of Psychiatry and Clinical Psychobiology, University of Barcelona, P. Vall d'Hebron 171, 08035-Barcelona, Catalonia-Spain; Phone: +34 933125 048; Fax: +34 9340215

84; cescera@ub.edu
\end{abstract}

Keywords: sensory memory; adaptation; event-related potentials (ERP); human; mismatch negativity (MMN); expectancy

Acknowledgements: This work was supported by the Spanish Ministry of Science and Innovation (PSI2009-08063; EUI2009-04086; Consolider-Ingenio2010 CSD2007-00012) and the Generalitat de Catalunya (2009SGR11). We would also like to thank David Costa Faidella for his support in the development of the experimental paradigm. 


\begin{abstract}
Single neurons in the primary auditory cortex of the cat show faster adaptation time constants to shortthan long-term stimulus history. This ability to encode the complex past auditory stimulation in multiple time scales would enable the auditory system to generate expectations of the incoming stimuli. Here, we tested whether large neural populations exhibit this ability as well, by recording human auditory evoked potentials (AEP) to pure tones in a sequence embedding short- and long-term aspects of stimulus history. Our results yielded dynamic amplitude modulations of the P2 AEP to stimulus repetition spanning from milliseconds to tens of seconds concurrently, as well as amplitude modulations of the mismatch negativity AEP to regularity violations. A simple linear model of expectancy accounting for both short- and long-term stimulus history described our results, paralleling the behavior of neurons in the primary auditory cortex.
\end{abstract}

Descriptors: Sensory memory, Event-related potentials (ERP), Stimulus-specific adaptation (SSA), Mismatch negativity (MMN), Expectancy

\title{
INTRODUCTION
}

Detecting unexpected sounds allows for prompt adaptive behav-ior to potentially relevant novel events. To accomplish that, the auditory system models the acoustic background forming sensory memory-traces, compares new input with inferences derived from the model, and elicits an error signal triggering an orienting attention mechanism whenever a sound mismatches the prediction (Escera \& Corral, 2007; Winkler, 2007). A relevant question, then, is how the auditory system forms the sensory memory traces used to model acoustic scenes. Recent evidence coming from animal single-cell recordings suggests that the main mechanism lies in the ability to match the neuron's spiking rate to stimulus statistics in multiple time scales (Ulanovsky, Las, Farkas, \& Nelken, 2004). In other words, neurons in the primary auditory cortex are sensitive to both local (short-term) and global (long-term) aspects of stimulus history simultaneously, a property that may aid us to capture the complexity of past auditory stimulation. Whether this neural mechanism generalizes to the activity elicited by large neural populations, as recorded in human electroencephalography (EEG), still remains to be determined.

Deviance detection in the auditory modality has been studied using the oddball paradigm, where a repeated sound (termed standard stimulus) is occasionally replaced by a rare sound (termed deviant stimulus). Evidence for deviance detection in the human auditory system comes traditionally from the mismatch negativity (MMN) auditory evoked potential (AEP; Na“a” ta“nen, Paavilainen, Rinne, \& Alho, 2007), which is isolated as the differential brain response to the deviant stimulus as compared (Näätänen, Gaillard,\& Mantysalo,1978). Evidence at the single-neuron level comes from animal stimulus-specific adaptation (SSA) studies. SSA, that is, the reduction of spiking rate to standard stimuli while keeping robust responses to deviant stimuli, has been found in primary auditory cortex (PAC) neurons (Ulanovsky, Las, \& Nelken, 2003; Ulanovsky et al., 2004) as well as in subcortical structures (Anderson, Christianson, \& Linden, 2009; Antunes, Covey, \& Malmierca, 2009; Malmierca, Cristaudo, Pérez-González, \& Covey, 2009; Pérez-González, Malmierca, \& Covey, 2005; Reches \& Gutfreund, 2008).

A striking property of SSA is that it matches stimulus statistics in multiple time scales simultaneously, showing fast adaptation time constants to short stimulus sequences, and slower adaptation time constants to long stimulus sequences (Ulanovsky et al., 2004). The encoding of stimulus probabilities in a wide temporal range would enable the auditory system to generate expectations of the incoming stimulation, which are crucial in the formation of auditory objects that typically have their features distributed over time (Bregman, 1990; Nelken \& Bar-Yosef, 2008; Nelken, Fishbach, Las, Ulanovsky, \& Farkas, 2003). Since SSA is a pervasive property of neurons along the auditory pathway, it is 
reasonable to expect a dynamic adaptation of human neural responses to repeated stimuli as measured with AEPs. Indeed, when the acoustic stimulation consists of trains of repetitive tones with tone frequency changing across trains (i.e., roving standard paradigm), the response to the standard stimulus changes gradually towards a positive deflection. This deflection, involving an increase of the P50 and P2 AEP amplitudes together with a decrease of the N1 AEP amplitude riding on a slow positive wave, has recently been isolated as the Repetition Positivity (RP; Baldeweg, Klugman, Gruzelier, \& Hirsch, 2004; Baldeweg, Wong, \& Stephan, 2006; Haenschel, Vernon, Dwivedi, Gruzelier, \& Baldeweg, 2005). Thus, RP has been proposed as the human AEP correlate of auditory sensory memory trace formation. Although several studies reported a fast development (Baldeweg et al., 2004, 2006; Haenschel et al., 2005) as well as a long-term persistence of the sensory memory-trace, from tens of seconds (Cowan, Winkler, Teder, \& Näätänen, 1993; Ritter, Sussman, Molholm, \& Foxe, 2002), up to minutes (Baldeweg, Williams, \& Gruzelier, 1999) and even days (Atienza, Cantero, \& Dominguez-Marin, 2002), no study has shown dynamic changes of AEPs to repetition in multiple time scales simultaneously. Here we explored the dynamics of adaptation of MMN and RP in an oddball sequence that embedded short- and long-term stimulus history, testing the hypothesis that MMN and RP amp amplitudes would be modulated in multiple time scales simultaneously. A simple linear model defining expectancy as a combination of both local and global aspects of stimulation history was devised to describe our results.

A striking property of these so-called novelty neurons is that SSA matches stimulus statistics in multiple time-scales (Ulanovsky et al., 2004), revealing an important role in the formation of auditory objects (Bregman, 1990; Nelken, Fishbach, Las, Ulanovsky, \& Farkas, 2003; Nelken \& Bar-Yosef, 2008). Since SSA is a pervasive property of neurons along the auditory pathway, it is reasonable to expect a dynamic adaptation of human neural responses to repeated stimuli as measured with AEPs. Indeed, modulations of MMN amplitude with repetition (Imada, Hari, Loveless, McEvoy, \& Sams, 1993; Javitt, Grochowski, Shelley, \& Ritter, 1998; Matuoka, Yabe, Ren, Hara, \& Kaneko, 2008; Sams, Alho, \& Näätänen, 1983) are primarily due to a gradual change in the response to the standard stimulus, which has recently been termed Repetition Positivity (RP; Baldeweg, Klugman, Gruzelier, \& Hirsch, 2004; Baldeweg, Wong, \& Stephan, 2006; Haenschel, Vernon, Dwivedi, Gruzelier, \& Baldeweg, 2005). However, no study has shown dynamic changes of AEPs to repetition in multiple time-scales. Here we explored the dynamics of adaptation of MMN and RP to stimuli in an oddball sequence tapping multiple implicit regularities, testing the hypothesis that MMN and RP amplitudes would be modulated in multiple time-scales simultaneously. A simple linear model accounting for both local and global aspects of stimulation history was devised to describe our results.

\section{MATERIALS AND METHODS Participants}

Twenty healthy volunteers ( 9 male, aged 18-28 years, mean age 21.4 years; one left handed) with no history of neurological, psychiatric or hearing impairment and with normal or corrected-to- normal visual acuity participated in the experiment. Subjects gave informed consent and received monetary compensation for their participation. The study was approved by the Ethics Committee of the University of Barcelona, according to the Code of Ethics of the World Medical Association (Declaration of Helsinki). Participants were asked to avoid smoking at least one hour before the experimental session as acute nicotine administration could affect RP amplitude (Baldeweg et al., 2006). All subjects underwent an audiometric test assessing the individual hearing level for tone frequencies equal to 401, 1409, and $3089 \mathrm{~Hz}$. Subjects showed no hearing threshold differences of 15

$\mathrm{dB}$ or more between the left and the right ear. Data of one subject had to be excluded from the analysis due to poor signal-to-noise ratio (i.e., less than $50 \%$ of artifact- free epochs in one block). 


\section{Stimuli and procedure}

The auditory stimuli consisted of pure sinusoidal tones of $40 \mathrm{~ms}$ duration including a $5 \mathrm{~ms}$ rise and a 20 ms fall time. They were generated with the Neurosoft (El Paso, TX, USA) sound program and delivered binaurally through headphones (Sennheiser HD-555, Wennebostel, Germany) by the Stim interface system (NeuroScan Labs, Sterling, VA). Tone intensity was individually adjusted to $55 \mathrm{~dB}$ sensation level (SL) with respect to the averaged hearing threshold for the three frequencies used in the audiometric test. The experiment used a switching fixed oddball sequence (Ulanovsky et al., 2004), comprised of two stimuli differing in tone frequency, which embedded multiple temporal scales of stimulation history (Fig.1.). In short, it consists of a repeating sequence of two stimuli differing in tone frequency appearing at fixed positions, designed in order to reveal shortand long-term adaptation effects. We defined these multiple time scales of stimulation, from the shortest to the longest, as follows: (1) "Repetition," consisting of consecutive trains of 2 (626 ms), 6 (1.9 s), and 12 (3.8 s) presentations of $\mathrm{f} 1$ stimulus (acting as the standard stimulus), each train followed by an $\mathrm{f} 2$ stimulus (acting as the rare or deviant stimulus); (2) "Run," consisting of two identical and consecutive presentations of a "Repetition" microsequence (Run1, Run2), so that trains of 2, 6, and 12 presentations of f1 stimulus in Run2 were comparable with those in Run1, having all Run1 stimulation history (6.3 s); and (3) "Switch," consisting of the repetition of the two "Runs" structure, but switching standard and deviant stimulus roles between f1 and f2 (SW1, SW2).

Note that the first stimulus of a "Switch" had in fact the role of a deviant stimulus in the previous micro-sequence but simultaneously that of the first standard stimulus in the present microsequence, resembling a roving standard paradigm (Baldeweg et al., 2004; Baldeweg et al., 2006; Cowan, Winkler, Teder, \& Näätänen, 1993; Haenschel et al., 2005). The Switch time scale allowed us to directly test the influence of adaptation to a tone repetition when this tone appears as a rare stimulus, by comparing f2 stimuli acting as deviants in SW1 with f1 stimuli acting as deviants in SW2, with f1 stimuli having all SW1 stimulation history as standards, i.e., 40 repetitions, $12.5 \mathrm{~s}$. It could be argued that the fixed order of the 2-, 6-, and 12-stimulus long repetitive trains within the Runs confounds local with global effects. For instance, the 12th $\mathrm{f} 1$ stimulus in Run 2 could be considered as the 40th f1 repetition in the last 45 sounds. However, as shown by Ulanovsky and colleagues (2004), the discharge rate of an auditory cortex neuron exhibiting SSA to a tone (f1) increases when it appears after a rare or deviant tone (f2), meaning that the latter partially erases the memory trace of the former (what has been called one-trial or after-deviant effect). Thus, our design is suitable to study local sequence effects, which are differently influenced by the global structure of the sequence.

To control for stimulus acoustic differences, tone frequencies $\mathrm{f} 1$ and $\mathrm{f} 2$ were chosen from a pool of six different frequencies $(401,619,827,1021,1217$, and $1409 \mathrm{~Hz}$ ) in a way that all possible pairwise combinations were covered, resulting in 30 different frozen sequences of 90 stimuli each. Although the perceptual difference between higher tones was smaller than between lower tones, the pairwise combinations eliminated all possible artifacts in the N1/MMN elicited to deviant stimuli. In order to enable stimulus comparisons between this study and single neuron SSA studies, we provide the normalized frequency difference (Df) between a pair of tones. Df, defined as (f2 - f1)/(f2 f1) $1 / 2$ according to single-cell recordings in animals (Ulanovsky et al., 2003), was 0.59 on average (SD50.36). Stimulus onset asynchrony (SOA) and inter-sequence interval were $313 \mathrm{~ms}$. The 30 frozen sequences were delivered in a pseudo-random order forming one single experimental block, with the constraint that a particular sequence could not contain any of the two frequencies appearing in the previous one, so that every repetition of the sequence would be treated as "new" by the neural populations encoding the frequencies of both tones. Four single blocks were presented separated by pauses of approximately $5 \mathrm{~min}$. In short, this complex design aimed to extract the auditory evoked responses to each stimulus in a sequence according to its position, regardless of the frequency of the tones that constitute it.

In order to control for deviant stimuli N1 refractoriness effects, we adapted the control condition developed by Schröger and Wolff (1996). In short, this control condition allows obtaining an 
AEP elicited by a stimulus with the same physical properties and probability as a deviant stimulus in an oddball sequence, embedded in a non-regular context. Thus, a "true" index of regularity violation can be extracted by comparing deviants against control stimuli.We presented two control blocks consisting of 60 frozen control sequences each. In these control sequences, control stimuli appeared in the same position and had the same particular frequencies as deviant stimuli in the oddball sequences. However, standard tones were replaced by random frequency tones (39 different frequencies, one per standard, ranging from 421 to $3089 \mathrm{~Hz}$; average Df between control tones and random tones, 0.82; SD50.56). It should be noted that the frequency range of the control stimuli and the average Df exceed those of the oddball stimuli. This means that a control stimulus (of the same tone frequency as the oddball stimulus) will be preceded by a tone that is, on average, more different in frequency than the one in the oddball sequence. Thus, control stimuli were expected to elicit larger or equal N1 amplitudes than deviant stimuli, excluding N1 refractoriness effects. Because no frequency repetition existed, control sequences presented the same structure as one single oddball switch of 45 stimuli (i.e., half of the frozen oddball sequence).

Participants sat in a comfortable chair in a sound-attenuated and electrically shielded room. They were instructed to ignore the sounds and watch a silent movie with subtitles. The first block in the experiment was a control block, followed by the four experimental blocks and a final control block. The total duration of the six blocks was 90 minutes approximately.

\section{Auditory evoked potentials recording and analysis}

The electroencephalogram (EEG) was continuously recorded with frequency limits of 0.05-100 Hz and digitized at a sampling rate of $500 \mathrm{~Hz}$ by a SynAmps amplifier (NeuroScan Inc., Herndon, VA). Pure tin electrodes were used for the EEG acquisition, 6 of which were mounted in a nylon cap (Electro-Cap International, Eaton, $\mathrm{OH}$ ) at the standard locations $\mathrm{F} 3, \mathrm{Fz}, \mathrm{F} 4, \mathrm{C} 3, \mathrm{Cz}$, and $\mathrm{C} 4$ according to the international 10-20 system. Additionally, two electrodes were positioned over the left and the right mastoids (M1 and M2). Vertical and horizontal electrooculogram (EOG) were measured from monopolar electrodes placed below (VEOG) and laterally (HEOG) to the right eye. The ground electrode was placed on the central forehead and the common reference electrode was attached to the tip of the nose. All impedances were kept below $5 \mathrm{k} \Omega$ during the whole recording session. Data were bandpass-filtered off-line between 0.3 and $20 \mathrm{~Hz}$ and averaged for epochs of $413 \mathrm{~ms}$ duration including a pre-stimulus baseline of $100 \mathrm{~ms}$. Epochs with a signal range exceeding $80 \mu \mathrm{V}$ at any EEG or EOG channel were excluded from the average.

Epochs used in the analysis of the effects of multiple time-scales of stimulation history on brain potentials were averaged separately for deviant events after 2, 6 and 12 standard events, for both "Runs" in both "Switches" (resulting in $3 \times 2 \times 2=12$ conditions), as well as for the standards preceding a deviant (12 conditions), and for control events after 2, 6 and 12 random frequency stimuli for both "Runs" (6 conditions). After rejection, a mean of 110 epochs (SD = 8.82; 74 minimum) were averaged for each stimulus type, condition and subject. In order to avoid possible deviant stimulus refractoriness effects, MMN difference waveforms were obtained by subtracting the brain potentials evoked by control stimuli from those evoked by deviant stimuli (Schröger \& Wolff, 1996). Peak latencies of mismatch potentials were determined from the Fz electrode as the largest negative peak in the interval of 80-180 ms for all difference waves and subjects separately. MMN mean amplitudes were derived in a $20 \mathrm{~ms}$ time window centered on the mean peak latency of the grand-average waveforms for all the 12 conditions (135-155 ms). Repetition effects on standard stimuli were assessed at Fz electrode by means of RP mean amplitudes, measured in a latency window ranging from 80 to $180 \mathrm{~ms}$ following the sound onset (Haenschel et al., 2005), and also by retrieving the mean amplitude in the latency window of the MMN (135-155 ms). Epochs used to fit exponential curves to determine the time course of adaptation of AEPs to standard events were averaged separately for all $\mathrm{f} 1 \mathrm{stimuli}$ in Switch1 and all f2 stimuli in Switch2 according to their position, except for deviant repetition or after- 
deviant events (see below). Epochs used to model brain potentials as a function of stimulus expectancy were averaged separately for all the 90 stimuli appearing in the sequence according to their position.

\section{Statistical analysis}

The effects on MMN peak latencies and mean amplitudes, as well as the effects on standard stimulus mean amplitudes in RP andMMNtime windows (50-250ms; 135-155 ms, respectively), Adaptation in the human auditory system 3 were evaluated with separate repeated measures analyses of variance (ANOVAs) including three factors: Switch $(1,2) \quad$ Run $(1,2) \quad$ Repetition $(2,6,12)$. Subsequent repeated measures ANOVAs were performed to assess interaction effects. The Greenhouse-Geisser correction was applied when appropriate. To characterize the time course of AEPs adaptation to standard stimuli, we retrieved the mean amplitudes in the P2 time window from the averaged AEPs across subjects (in order to isolate better the obtained repetition effects, which inverted their polarity at the mastoid electrodes, Fz was re-referenced to M1) and performed a nonlinear least-square fit to find the best-fitting exponential function as follows: decay size $x \quad\left(1-e^{t / \tau}\right)+$ asymptote.

\section{Modeling auditory evoked potentials as a function of stimulus expectancy}

A simple linear model was devised in order to account for brain potential modulations as a function of stimulus expectancy. Mean amplitudes of responses to all standard and deviant stimuli appearing in the sequence (90 stimuli; epochs averaged according to their position) were retrieved in the MMN/P2 latency window for all subjects at the Fz electrode re-referenced to M1. Assuming that negative brain potential values in that time window decrease with increasing number of repetitions/higher probability (Imada, Hari, Loveless, McEvoy, \& Sams, 1993; Javitt, Grochowski, Shelley, \& Ritter, 1998; Sams, Alho, \& Näätänen, 1983), we defined stimulus expectancy as a linear combination of two independent factors: (1) the memory for the local stimulus history (M); and (2) the estimated probability of the stimulus (P). For M, we postulated that the local effect of preceding stimuli on the expectancy of the current stimulus is an exponentially decaying function of serial position (Squires, Wickens, Squires, \& Donchin, 1976; Ulanovsky et al., 2004). In particular, the memory M for stimulus k (i.e., f1 or f2) at position $\mathrm{N}$ as a function of the sequence of past stimuli $\mathrm{Si}$ is assumed to be:

$$
M_{k N}=\frac{1}{Z} \sum_{i=N-1}^{N-m} \alpha^{N-i} S_{i}
$$

with Si taking the value of 1 when the stimulus at position $\mathrm{i}$ equals $\mathrm{k}$ and 0 when the stimulus at position $\mathrm{i}$ is unequal to $\mathrm{k}$ (i.e., in order to model the memory for an f1 stimulus we only take into account previous $\mathrm{f} 1$ stimuli); $\mathrm{m}$ is the number of past stimuli conforming the local sequence (here, as in Squires et al., 1976; and Ulanovsky et al., 2004, $\mathrm{m}=5$ ) and the constant a determines the time course of memory decay $(0 \leq \alpha \leq 1)$. $\mathrm{Z}$ is a normalization factor that takes the maximum value of $\mathrm{M}$, so that $0 \leq$ $\mathrm{M} \leq 1$ (Ulanovsky et al., 2004), defined as:

$$
Z=\sum_{i=1}^{m} \alpha^{i}
$$

The second factor (P) was modelled taking into account how the "subjective probability" of a stimulus is represented and updated over time, rather than how it changes on average (Mars et al., 2008). It should be noted that in the "frozen oddball sequence" used in the present experiment all stimuli are, globally, equiprobable. Thus, global probability could not be used as a factor as it has been done in previous studies using random oddball paradigms (Squires et al., 1976; Ulanovsky et al., 2004). Instead, we used the estimate probability (P) of a stimulus ( 11 or f2) appearing in the sequence, which is continuously modified by the occurrence of new stimuli. Because the estimate probability pk will be 
0 if the stimulus $\mathrm{k}$ has not been previously presented, an a priori probability is needed in order to assume initially that all stimuli are equally likely to occur. This issue was solved by using a prior Dirichlet distribution (Mars et al., 2008). A uniform Dirichlet distribution is parameterized by a vector $\gamma$ $=[\gamma 1, \ldots \gamma \mathrm{k}]$ of dimension equal to the number of possible elements, and written as $\mathrm{P}(\mathrm{p} \mid \gamma)=\operatorname{Dir}(\mathrm{p} ; \gamma \mathrm{k})$. Choosing all elements of $\gamma$ equal to one means to start with a sequence of equiprobable stimuli. In the present case, using six different frequencies in the experimental blocks results in an a priori probability for a stimulus of $1 / 6 \approx 0.17$. The subsequent distribution representing the estimated probabilities after $\mathrm{j}$ trials, $\mathrm{X} \mathrm{j}$, is given by

$$
P\left(p \mid X^{j}, \gamma\right)=\operatorname{Dir}\left(p^{j} ; n_{k}^{j}+\gamma_{k}\right)
$$

where $n_{k}^{j}$ refers to the number of occurrences of stimulus $k$ up to position $\mathrm{j}$. This distribution takes again a Dirichlet form, parametrized by the vector with elements equal to $n_{k}^{j}+\gamma_{k}$. In short, this expression states that the estimated probability of finding a particular stimulus $\mathrm{k}$ in position $\mathrm{j}$ is determined by the sequence of stimuli presented and by the a priori probabilities (parametrized by $\gamma$ ). The expression that represents the probability of observing stimulus $\mathrm{k}$ in position $\mathrm{j}$ as a function of the estimated probabilities in position $\mathrm{j}-1$

$$
\begin{gathered}
p\left(x^{j}=k \mid X^{j-1}, \gamma\right)=\frac{n_{k}^{j-1}+1}{N^{j-1}+K}=\tilde{p}_{k}^{j} \\
N^{j-1}=\sum_{k=1}^{K} n_{k}^{j-1}
\end{gathered}
$$

is the total number of stimuli preceding position $\mathrm{j}$, which is equal to $\mathrm{j}-1$, and $\mathrm{K}$ stands for the number of possible stimuli $(\mathrm{k}=6)$. To sum up, the prediction of the probability of observing stimulus $\mathrm{k}$ on position $\mathrm{j}\left(\sim_{\mathrm{p}}^{\mathrm{j}}{ }_{\mathrm{k}}\right)$ depends on all preceding observations and a uniform prior.

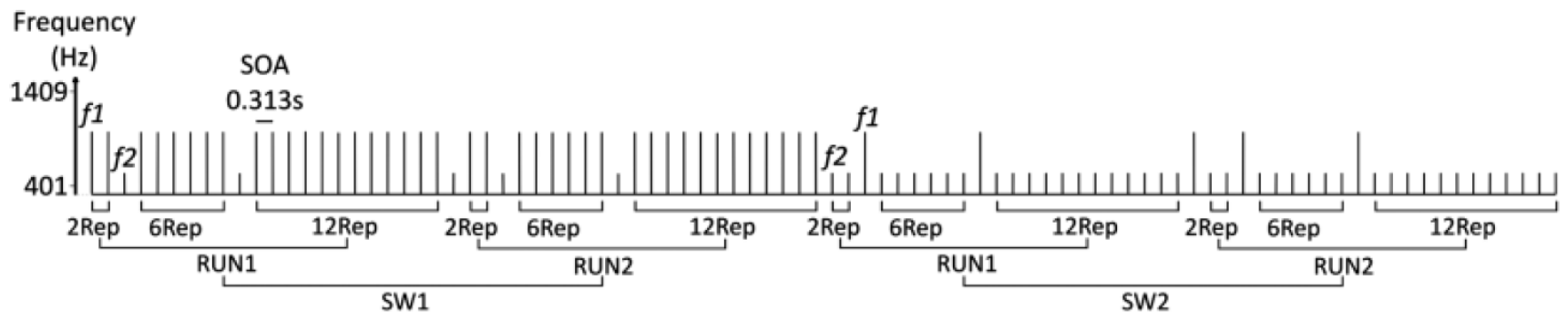

Figure 1. Schematic diagram of the stimulus sequence. A tone of frequency f1 was presented in a row of 2, 6, and 12 consecutive stimuli, each of which was followed by a tone of frequency f2. This micro sequence was presented in two successive runs, which we called Run1 and Run2. Both Runs formed the first half of the sequence, called Switch1, where f1 acted as a standard stimulus and $\mathrm{f} 2$ as a deviant stimulus. Switch2 had the same structure as Switch1, but frequency roles were switched so that $\mathrm{f} 1$ acted as a deviant stimulus and $\mathrm{f} 2$ as a standard stimulus. The frozen sequence was preceded and followed by equal sequences containing stimuli of a different pair of frequencies (ranging from 401 to $1409 \mathrm{~Hz}$ ). SOA, Stimulus Onset Asynchrony. 
Finally, stimulus expectancy was defined as a linear combination of the memory for the local stimulus history $(\mathrm{M})$ and the estimate probability of the stimulus $(\mathrm{P})$, as shown in the following expression: $\mathrm{A}=\mathrm{aM}+\mathrm{bP}+\mathrm{c}$, where $\mathrm{a}, \mathrm{b}$, and $\mathrm{c}$ are the parameters to be adjusted in a multiple linear regression analysis, and $\mathrm{A}$ is the predicted amplitude value of the brain potential. We modeled the amplitudes of all 90 stimuli averaged across subjects and for each subject individually.

\section{Results}

Grand-average waveforms evoked to standard (gray), deviant (black), and control (dotted trace) stimuli are illustrated for each condition in Figure 2, together with deviant-minus-control waveforms (Switch1, black thick trace; Switch2, gray thick trace). RP can be identified as a repetition-enhanced positive slow wave evoked to standard stimuli that develops drastically from 2 to 6 repetitions in Run1 (both Switches). Embedded in this RP, we can observe the emergence of the P2 potential increasing with the number of repetitions in a time range coinciding with that of the MMN, which increased as well the more standard stimuli preceded a deviant stimulus. Furthermore, a remarkable decrease in the amplitudes of the MMNs elicited to deviant stimuli in Switch2 can be seen in comparison to those elicited to deviant stimuli in Switch1.

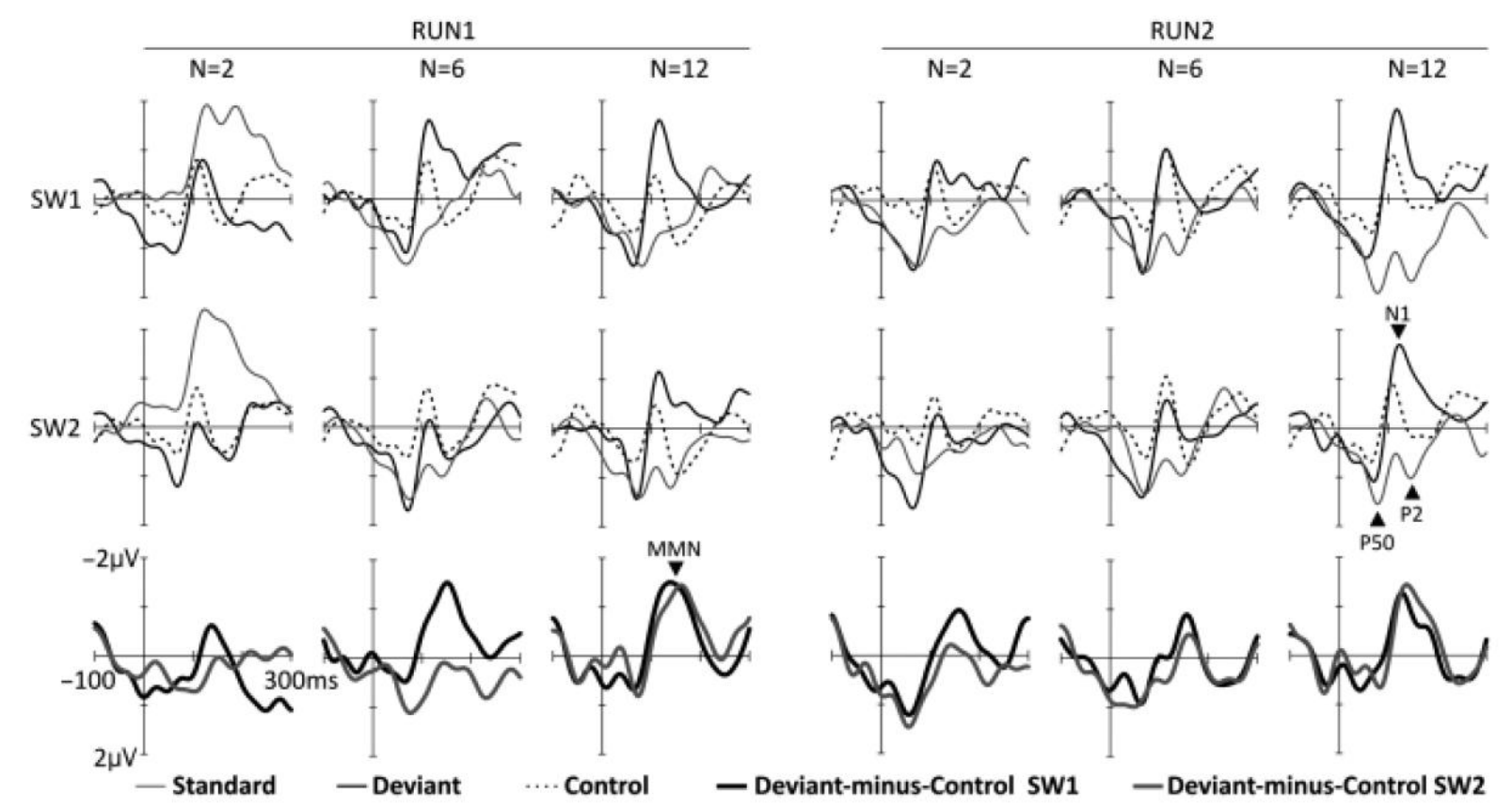

Figure 2. Grand-average waveforms for standard (gray), deviant (black), and control (dotted trace) stimuli after 2, 6, and 12 stimulus presentations in both Runs and Switches, together with deviant-minus-control difference waves (SW1, black thick trace; SW2, gray thick trace). The arrows point to the P50, N1, and P2 AEP components in the grand-average waveforms as well as to the MMN in the difference waveforms. 
Sensory memory-trace formation to repeated stimuli, as indexed by amplitude changes of RP, was strengthened in a short-term time scale only to the first presentations of a given acoustic stimulus (Run1), thus showing a fast adaptation of the neural response that reached the maximum after 12 consecutive stimulus presentations (interaction between Run and Repetition factors, $\mathrm{F}(2,36)=16.895$, $\mathrm{p}<.001, \mathrm{y} 2=0.484$; a post-hoc repeated measures ANOVA revealed significant effects of Repetition in Run1, $\mathrm{F}(2,36)=26.430, \mathrm{e}=0.665, \mathrm{p}<.001, \mathrm{y} 2=0.595$, but no significant effects were obtained in Run2, $\mathrm{Z} 250.076)$. However, the P2 potential evoked to repeated sounds revealed a better sensitivity to multiple time scales of stimulus history than the RP, showing a marked increase with the number of repetitions in the first presentations of a given tone (Run1) together with a slighter increase in further presentations (Run2; interaction between Run and Repetition factors, $F(2,36)=15.916, p<.001$, y2=0.469; a post-hoc repeated measures ANOVA revealed significant effects of Repetition in Run1, $\mathrm{F}(2,36)=38.453$, e $=0.682, \mathrm{p}<.001, \mathrm{p} 2=0.681$, and in $\mathrm{Run} 2, \mathrm{~F}(2,36)=3.285, \mathrm{p}<.05, \mathrm{p} 2=0.154)$. Previous presentation of a tone as a deviant stimulus exerted no effect on brain potentials evoked to the same acoustic stimulus when occurring as a standard stimulus (no significant Switch effect in RP or P2). P2 mean amplitudes evoked to standard stimuli are shown in Figure 3. As no effects or interactions including the factor Switch were found, data were pooled across the two Switches for graphic purposes. When fitting exponential functions, the time course of adaptation of brain potentials to repeated stimuli was similar for standard stimuli in Switch1 $(\mathrm{t}=10.4 \mathrm{~s}$, with $95 \%$ confidence bounds; $\mathrm{R}^{2}$ (adjusted) $\left.=0.536\right)$ and Switch $\left(\mathrm{t}=8.2 \mathrm{~s}\right.$, with $95 \%$ confidence bounds; $\mathrm{R}^{2}$ (adjusted) $\left.=0.466\right)$ (Figure 4; black, exponential curve fits for f1 (white) in Switch1 and f2 (gray) in Switch2).

\section{Effects of Multiple Time Scales of Stimulus History on Deviance Detection}

True memory-based deviance detection, as indexed by the amplitude of the MMN controlled for refractoriness effects, was enhanced in a short-term time scale as a function of the number of standard stimuli preceding the deviant stimulus (Repetition effect, $\mathrm{F}(2,36)=4.320, \mathrm{p}<.05, \mathrm{y} 2=50.194$; from 2 [0.6s] to 12 stimulus presentations [4s]). This enhancement was found together with a marked decrease of MMN when the same acoustic stimulus deviating from the local sequence was previously presented as a standard stimulus (Switch effect, $\mathrm{F}(1,18)=6.050, \mathrm{p}<.05, \mathrm{y} 2=0.252$ ), thus reflecting long-term effects of stimulation

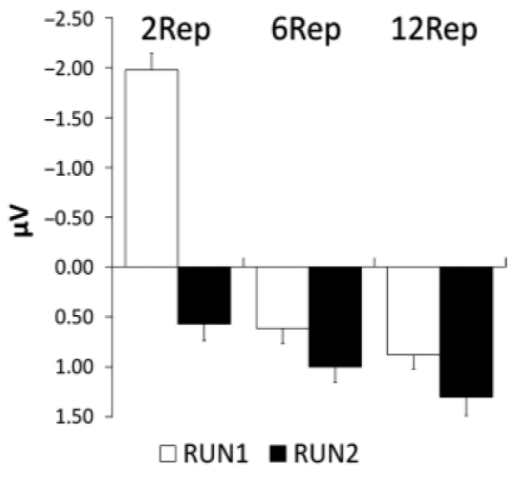

Figure 3. P2 mean amplitudes elicited to standard stimuli after 2, 6, and 12 stimulus repetitions for Run1 (white) and Run2 (black) for averaged Switches (135-155 ms time window; amplitudes in $\mathrm{mV}$; error bars denote standard error of means). P2 mean amplitudes increased the more a standard stimulus was repeated, at both short- (Repetition) and longterm (Run) time scales. 


\section{Time (s)}

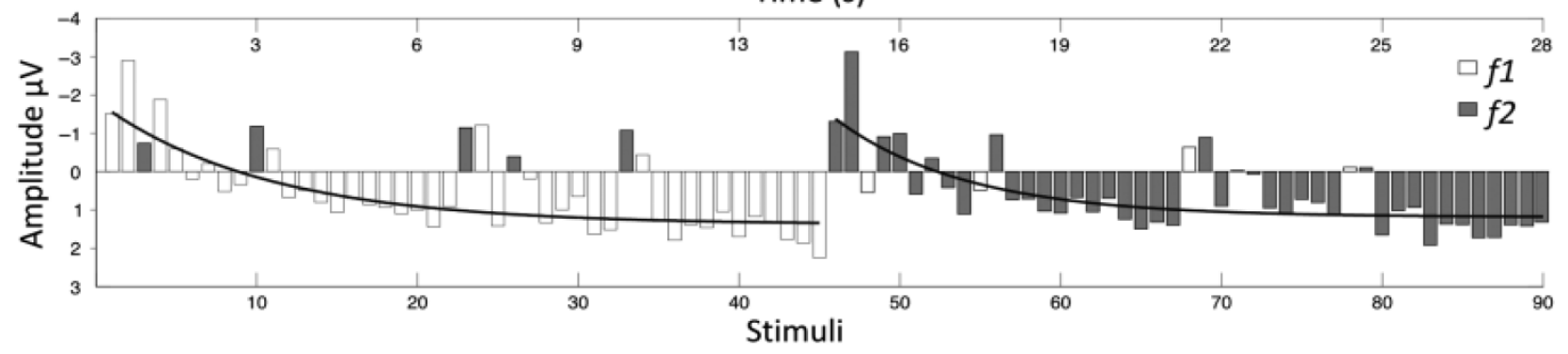

Figure 4. Amplitude of brain potentials in the MMN/P2 timewindow obtained for all 90 stimuli appearing in the sequence together with the exponential fits for $\mathrm{f} 1$ in Switch1 and $\mathrm{f} 2$ in Switch2 (amplitudes in $\mathrm{mV}$; f1 stimulus, white; f2 stimulus, gray; exponential curve fit for Switch1and Switch2, black).

history ( 10.33 seconds). No modulations were found for MMN peak latencies, with a mean across conditions of $145 \mathrm{~ms}$ following stimulus onset. MMN mean amplitudes are shown in Figure 5. As no effects or interactions including the factor Run were found, data were pooled across the two Runs for graphic purposes. AEP Amplitude as a Function of Stimulus Expectancy Brain potentials amplitudes evoked to all 90 stimuli appearing in the sequence in the MMN/P2 time range are illustrated in Figure 4. We first determined the memory decay constant a that maximized the linear relationship between brain potentials and the memory for the local stimulus history M. The obtained value was $a=0.786$, which determined a time constant of memory decay (i.e., time for the memory-trace to decay to the half of its value) of: $\tau_{\mathrm{M}}=1 /(1-$ a) 4.67 stimuli $1.46 \mathrm{~s}$ (Ulanovsky et al., 2004). The estimated probability of a stimulus (P) was calculated for each of the 90 stimuli in the sequence (see Materials and Methods). We then performed a multiple linear regression analysis that determined the equation relating the amplitude measures to $\mathrm{M}$ and $\mathrm{P}$ factors, resulting in the following expression: $\mathrm{A}=2.1081 \mathrm{M}+1.3235 \mathrm{P}-$ 1.6683. Brain potential observed amplitudes evoked to all 90 stimuli are plotted in Figure 6 as a function of stimulus expectancy. A positive correlation value of $\mathrm{R}=0.764$ and a significant model adjustment of $\mathrm{R}^{2}$ (adjusted) $=0.579, \mathrm{~F}(1,89)=123.258 ; \mathrm{p}<10^{-17}$, indicated that brain potential amplitude in the MMN/P2 time window increased linearly as a function of stimulus expectancy. A stepwise method used to compute single-variable regressions revealed that each single parameter in the model could itself explain a significant amount of the variance in the data: $M: R^{2}$ (adjusted) $=0.515$, $\mathrm{F}(1,89)=95.579, \mathrm{p}<10$ 14; $\mathrm{P}: \mathrm{R}^{2}$ (adjusted) $=0.251, \mathrm{~F}(1,89)=30.784, \mathrm{p}<10 \quad 6$. Moreover, the linear model provided a significant fit for each individual subject as well: mean $R^{2}$ (adjusted) $=0.268$; $\mathrm{SD}=0.183$; mean $\mathrm{p}$ values $<10 \quad 18 ; \mathrm{SD}=0.05$.

\section{Discussion}

The present data shows dynamic amplitude modulations of AEP to simple sounds spanning multiple time scales concurrently, paralleling SSA properties. AEP amplitude modulations were seen as a linear increase of positivity in the time range of the $\mathrm{P} 2$ potential, coinciding with that of the MMN, as a function of stimulus expectancy. Thus, our results suggest that adaptation in multiple time scales is a basic property of the auditory system expanding fromthe single-neuron scale to a wider range of neural populations. 


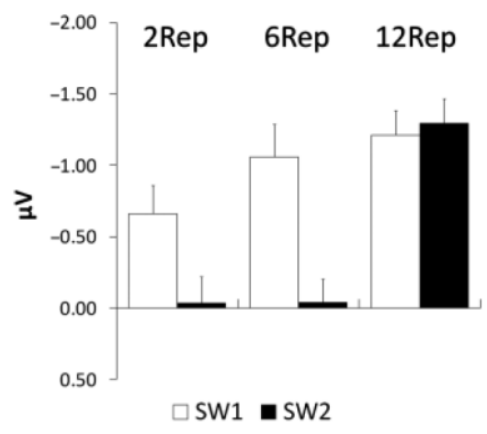

Figure 5. MMN mean amplitudes after 2, 6, and 12 standard stimuli in Switch1 (white) and Switch2 (black) for averaged Runs (135-155 ms time window; amplitudes in mV; error bars denote standard error of means). MMNwas significantly affected by short- and long-termstimulus history simultaneously, increasing in amplitude the more a preceding standard stimulus was repeated (Repetition effect) and being suppressed when deviant stimuli featured a frequency previously presented as a standard stimulus (Switch effect).

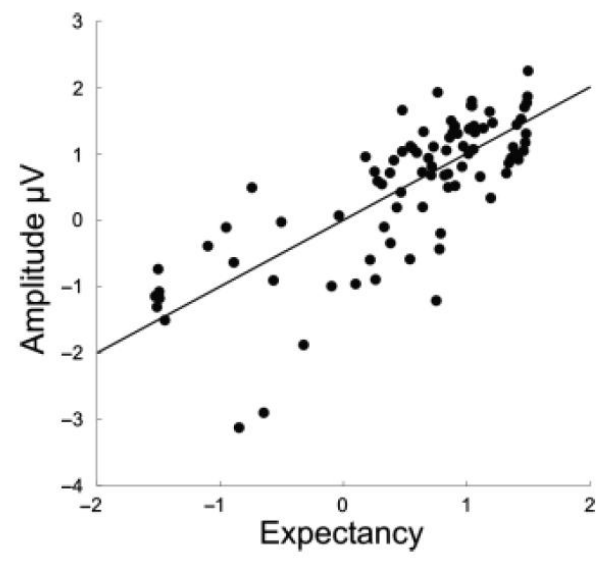

Figure 6. Brain potential observed amplitudes to all 90 stimuli appearing in the sequence plotted as a function of the predicted expectancy score (amplitudes in $\mathrm{mV}$; expectancy values in arbitrary units). Note the linear increase of the AEP amplitude in the MMN/P2 time window as a function of stimulus expectancy. 


\section{Multiple time-scales of adaptation in the human auditory system}

Previous studies examining the effect of stimulus history on human auditory sensory memory reported a fast development (Baldeweg et al., 2004; Baldeweg et al., 2006; Haenschel et al., 2005) as well as a long-term persistence of the sensory memory-trace, from tens of seconds (Cowan et al., 1993; Ritter, Sussman, Molholm, \& Foxe, 2002), up to minutes (Baldeweg, Williams, \& Gruzelier, 1999) and even days (Atienza, Cantero, \& Dominguez-Marin, 2002).

Here we used a design from a single-neuron recording study (Ulanovsky et al., 2004) allowing us to reveal that human AEPs to repeated stimuli adapt in multiple time scales simultaneously. Specifically, we showed a fast adaptation time constant to the local sequence preceding the stimulus $\left(\begin{array}{lll}\tau_{\mathrm{M}} & \sim 1.5 \mathrm{~s}\end{array}\right)$ concurrently with a slower adaptation time constant involving a longer history of stimulation $(\tau \sim 10 \mathrm{~s})$. This adaptation lasted $10 \mathrm{~s}$, as seen by the decrease of the neural response to a deviant stimulus that has been preceded by several repetitions of the same acoustic stimulus (i.e., switch effect). This slow recovery coincides with the estimate duration of sensory memory as seen by AEPs (Bottcher-Gandor \& Ullsperger, 1992) and behavioral studies (Cowan, 1984), and contrasts to the simultaneous fast recovery seen in the response to post-deviant stimuli, which show a partial reset of the sensory-memory trace ( $\sim 0.3$ s, one-trial effect; Sams, Alho, \& Näätänen, 1984). Unfortunately, longer time scales in the range of minutes as those obtained in SSA by Ulanovsky and colleagues (2004) couldn't be explored in this study because of the use of a roving standard paradigm across the oddball sequences. However, interestingly, the adaptation to the local sequence of stimulation developed in a similar time range to that reported in PAC neurons of the cat (Ulanovsky et al., 2004). Additionally, we succeeded to predict amplitude modulations of AEPs as a function of stimulus expectancy with a simple linear model accounting for both local and global aspects of stimulation history. Previous research linked stimulus predictability to amplitude and latency modulations of the P300 component of the cognitive evoked potentials, while subjects performed some task related to auditory or visual stimuli (Fogelson et al., 2009; Mars et al., 2008; Squires et al., 1976). The P300 has been related to the evaluation of inferences about the environment as a function of the context (Squires et al., 1976), attention switching (Escera, Alho, Schröger, \& Winkler, 2000) and learning of surprising events (Donchin, 1981). Interestingly, similar models could explain our results in human AEPs to unattended sounds as well as neuronal firing patterns in the PAC of anesthetized animals (Ulanovsky et al., 2004), suggesting that inference based on updating probabilities is a basic property of the auditory system not necessarily under the influence of top-down processes.

\section{Repetition Effects on Auditory Evoked Potentials}

Modulations of the AEP correlated with stimulus repetition were observed as a frontocentral positive waveform, between 50 and $250 \mathrm{~ms}$ post-stimulus, which we identified as RP (Baldeweg et al., 2004, 2006; Haenschel et al., 2005). It has been argued that RP does not consist of a unitary phenomenon but rather a combined modulation of P50, N1, and P2 potentials (Haenschel et al., 2005). In fact, our results showed better sensitivity to stimulation history when the amplitude of the AEP to standard stimuli was measured in the P2 potential, which largely coincides with the time range of the MMN AEP elicited to regularity violations ( $145 \mathrm{~ms}$ post-stimulus). Other studies reported P2 enhancements in time scales of minutes (Baldeweg et al., 1999) or days (Atienza et al., 2002), as well as N1 habituation (Butler, Spreng, \& Keidel, 1969; Näätänen et al., 1988; Picton,Woods, \& Proulx, 1978) to repeated stimuli. P50 enhancements have also been reported to repetitions embedded in constantly changing acoustic backgrounds (Dyson, Alain, \& He, 2005). However, repetition usually diminishes P50 and P2 potentials (Boutros et al., 1995; Javitt, 2000; Lijffijt et al., 2009; Lu, Williamson, \& Kaufman, 1992), meaning that refractoriness effects should in turn diminish RP. These differences between studies could arise from the use of different experimental paradigms, such as oddball, roving standard, or pairedclick paradigms, as well as from differences in data analysis. Indeed, P50 studies usually exclude the contribution of low frequency-band activity included in MMN and RP studies (from 0.1 to $10 \mathrm{~Hz}$ ). 
Hence, we tested additionally whether P50 enhancements with repetition could be due to slow wave contributions by re-analyzing our data with the appropriate filter settings (10 to $49 \mathrm{~Hz}$; see Appendix I). We found a reduction in the P50 amplitude (70 ms post-stimulus) evoked to standard stimuli compared to deviant stimuli that was not modulated across conditions, and no differences between the P50 evoked to deviant and control stimuli were found. These results agree with previous research showing that P50 habituation fully develops within one repetition (Rosburg et al., 2004) and support the notion that the habituation of early AEPs to repetition possibly indexes the formation of stimulus feature traces and not an integrated stimulus representation (Näätänen \& Winkler, 1999). It also highlights the importance of low frequency bands in the development of RP, previously related to an oscillatory inference generation mechanism involving the encoding of temporal contingencies (Bendixen, Schrö ger, \& Winkler, 2009; Clementz, Barber, \& Dzau, 2002; Näätänen, 1992). We suggest that the development of this positive slow wave reflects the entrainment of neural populations encoding a certain frequency with rhythmical stimulation (Lakatos, Karmos, Mehta, Ulbert, \& Schroeder, 2008; Will \& Berg, 2007). This could explain why the RP starts well before stimulus onset, why it is not present in control stimuli, and why the response to the second stimulus at the beginning of each Switch presents a prominent negative response (by interpreting deviant stimuli as breaking the entrainment, which takes several tone repetitions to be reinstated). However, this hypothesis needs further testing in future experiments properly designed to perform time-frequency analyses of the EEG data.

\section{Neural Mechanisms of Deviance Detection in the Auditory System}

Since its discovery, the MMN has been considered an index of primitive intelligence in the auditory cortex (Näätänen, Tervaniemi, Sussman, Paavilainen, \& Winkler, 2001). Two main hypotheses compete for the interpretation of its underlying neural mechanisms: the regularity violation hypothesis, stating that $\mathrm{MMN}$ is generated by the mismatch between new input and predictions of future sensory events driven by a subset of extrapolatory sensory neurons (Näätänen, 1992); and the N1 adaptation hypothesis, proposing that MMN emerges when comparing an N1 response to a deviant stimulus with a refractory N1 response to a repeated stimulus (Jääskeläinen et al., 2004), both arising from the activity of tonotopically organized afferent sensory neurons subject to adaptation and lateral inhibition (May et al., 1999; May \& Tiitinen, 2010; for an extensive discussion about the concepts of neural adaptation and habituation in single-cell recordings and AEPs, please refer to Nelken \& Ulanovsky, 2007). Our results, as well as those arising from studies using similar control stimuli (Jacobsen \& Schröger, 2001, 2003; Jacobsen, Horenkamp, \& Schröger, 2003; Jacobsen, Schröger, Horenkamp, \& Winkler, 2003; Schröger \& Wolff, 1996), are in agreement with the position advanced by Näätänen (1992) by showing that the time course of the controlled MMN extends beyond that of the N1 potential. Moreover, we found an enhancement of the controlled MMN amplitude with the local sequence of stimulation, a result at odds with that reported by Haenschel and colleagues (2005), whereMMN amplitude incrementswith repetitionwere entirely due to changes in the standard AEP. While other studies reported MMN amplitude increments with the local sequence of stimulation (Giese-Davis, Miller, \& Knight, 1993; Sams et al., 1983) or lower deviant probabilities (Imada et al., 1993; Javitt et al., 1998), the nature of the MMN repetition effect is still controversial. For instance, none of these studies separated the differential contributions of the standard AEP repetition effect from those of the deviant; studies focused on the RP using roving standard paradigms report significant increments of the deviant negativity as well as standard positivity (Baldeweg et al., 2004, 2006), whereas Haenschel and colleagues (2005) only report increments in the standard positivity; and a study by Horvath, Winkler, and Bendixen (2008), which separated local sequences naturally occurring in an oddball paradigm with two equiprobable stimuli, only reported amplitude increments of the N1/MMN AEP elicited to deviant stimuli. A possible explanation for the differences between these studies is the fact that different stimulation paradigms lead to different results: roving, standard paradigms seem to enhance the changes to the standard AEP while oddball paradigms don't. In the present study, our findings suggest 
that, in addition to the adaptation to repetition in multiple time scales simultaneously, the system's excitability strengthens for stimuli differing from the repeated stimulus (Näätänen, 1992). Interestingly, an increase of the response to deviant stimuli in comparison to equiprobable control stimuli has also been shown in PAC neurons of the cat (Ulanovsky et al., 2003).

An intriguing finding observed here is the fact that $\mathrm{MMN}$ is reduced to deviant stimuli formed by a sound with a long history of stimulation. Simple adaptation cannot account for this reduction because it is reverted by local sequence effects and reinstated with only one presentation of the stimulus (Ritter et al., 2002), as seen by local (Repetition) but not global (Run) sequence effects in Switch2. We suggest that the adaptation of a subset of neurons in the auditory system, which encode stimulus probabilities inmultiple time scales and thus enable the system to generate expectations of the incoming stimulation, may account for the P2 enhancement as a gradual decrease of the MMN response. This explanation fits well with the predictive coding approach, which attempts to interpret sensory systems as predictive machines trying to infer and learn the causes of sensory data by minimizing prediction error (i.e., surprise), adjusting top-down predictions to bottom-up inputs in every hierarchical level through synaptic plasticity (Friston, 2005). Importantly, this perspective integrates both the regularity violation and the adaptation MMN generation hypotheses (Garrido, Kilner, Kiebel, \& Friston, 2009), interpreting MMN as an index of prediction error (Baldeweg, 2006, 2007). In summary, we have demonstrated that large neural populations exhibit the ability to match neural activity to stimulus statistics in multiple time scales, paralleling the behavior of PAC neurons. This wide range of adaptation time constants could be useful for supporting representations of auditory objects that typically have their features distributed over time (Nelken et al., 2003). Thus, the present results may help to establish a crucial bridge between human and animal research towards unraveling the neural mechanisms underlying acoustic background encoding.

\section{REFERENCES}

Anderson, L. A., Christianson, G. B., \& Linden, J. F. (2009). Stimulus-specific adaptation occurs in the auditory thalamus. The Journal of Neuroscience, 29(22), 7359-7363.

Antunes, F.M., Covey, E., \& Malmierca, M.S. (2009). Is there stimulus-specific adaptation in the auditory thalamus? In 'Advances in Auditory Physiology, Psychophysics and Models'. Ed by E.A. Lopez-Poveda, A.R. Palmer, and R. Meddis. Springer, New York. In press.

Atienza, M., Cantero, J. L., \& Dominguez-Marin, E. (2002). The time course of neural changes underlying auditory perceptual learning. Learning \& Memory (Cold Spring Harbor, N.Y.), 9(3), 138-150.

Baldeweg, T. (2007). ERP repetition effects and mismatch negativity generation: A predictive coding perspective. Journal of Psychophysiology, 21(3), 204-213.

Baldeweg, T., Williams, J. D., \& Gruzelier, J. H. (1999). Differential changes in frontal and subtemporal components of mismatch negativity. International Journal of Psychophysiology, 33(2), 143-148.

Baldeweg, T., Klugman, A., Gruzelier, J., \& Hirsch, S. R. (2004). Mismatch negativity potentials and cognitive impairment in schizophrenia. Schizophrenia Research, 69(2-3), 203-217.

Baldeweg, T., Wong, D., \& Stephan, K. E. (2006). Nicotinic modulation of human auditory sensory memory: Evidence from mismatch negativity potentials. International Journal of Psychophysiology, 59(1), 49-58.

Bendixen, A., Schroger, E., \& Winkler, I. (2009). I heard that coming: Event-related potential evidence for stimulus-driven prediction in the auditory system. The Journal of Neuroscience, 29(26), 8447-8451.

Böttcher-Gandor, C., \& Ullsperger, P. (1992). Mismatch negativity in event-related potentials to auditory stimuli as a function of varying interstimulus interval. Psychophysiology, 29(5), 
546-550.

Boutros, N. N., Torello, M. W., Barker, B. A., Tueting, P. A., Wu, S. C., \& Nasrallah, H. A. (1995). The P50 evoked potential component and mismatch detection in normal volunteers: Implications for the study of sensory gating. Psychiatry Research, 57(1), 83-88.

Bregman, A. S. (1990). Auditory scene analysis: The perceptual organization of sound. Cambridge, MA US: The MIT Press.

Butler, R. A., Spreng, M., \& Keidel, W. D. (1969). Stimulus repetition rate factors which influence the auditory evoked potential in man. Psychophysiology, 5(6), 665-672.

Clementz, B. A., Barber, S. K., \& Dzau, J. R. (2002). Knowledge of stimulus repetition affects the magnitude and spatial distribution of low-frequency event-related brain potentials. Audiology \& Neuro-Otology, 7(5), 303-314.

Cowan, N. (1984). On short and long auditory stores. Psychological Bulletin, 96(2), 341-370. Cowan, N., Winkler, I., Teder, W., \& Näätänen, R. (1993). Memory prerequisites of mismatch negativity in the auditory event-related potential (ERP). Journal of Experimental Psychology. Learning, Memory, and Cognition, 19(4), 909-921.

22Multiple time-scales of adaptation in the human auditory system

Donchin, E. (1981). Presidential address, 1980. surprise!...surprise? Psychophysiology, 18(5), 493-513.

Dyson, B. J., Alain, C., \& He, Y. (2005). I've heard it all before: Perceptual invariance represented by early cortical auditory-evoked responses. Brain Research.Cognitive Brain Research, 23(2-3), 457-460.

Escera, C., Alho, K., Schröger, E., \& Winkler, I. (2000). Involuntary attention and distractibility as evaluated with event-related brain potentials. Audiology \& Neuro-Otology, 5(3-4), 151-

166.

Escera, C., \& Corral, M. J. (2007). Role of mismatch negativity and novelty-P3 in involuntary auditory attention. Journal of Psychophysiology, 21(3-4), 251-264.

Fogelson, N., Wang, X., Lewis, J. B., Kishiyama, M. M., Ding, M., \& Knight, R. T. (2009). Multimodal effects of local context on target detection: Evidence from p3b. Journal of Cognitive Neuroscience, 21(9), 1680-1692.

Friston, K. (2005). A theory of cortical responses. Philosophical Transactions of the Royal Society of London.Series B, Biological Sciences, 360(1456), 815-836.

Garrido, M. I., Kilner, J. M., Kiebel, S. J., \& Friston, K. J. (2009). Dynamic causal modeling of the response to frequency deviants. Journal of Neurophysiology, 101(5), 2620-2631.

Haenschel, C., Vernon, D. J., Dwivedi, P., Gruzelier, J. H., \& Baldeweg, T. (2005). Eventrelated brain potential correlates of human auditory sensory memory-trace formation. The Journal of Neuroscience, 25(45), 10494-10501.

Horvath, J., Czigler, I., Sussman, E., \& Winkler, I. (2001). Simultaneously active pre-attentive representations of local and global rules for sound sequences in the human brain. Brain Research.Cognitive Brain Research, 12(1), 131-144.

Imada, T., Hari, R., Loveless, N., McEvoy, L., \& Sams, M. (1993). Determinants of the auditory mismatch response. Electroencephalography and Clinical Neurophysiology, 87(3), 144-153.

Jääskeläinen, I. P., Ahveninen, J., Bonmassar, G., Dale, A. M., Ilmoniemi, R. J., Levanen, S., et al. (2004). Human posterior auditory cortex gates novel sounds to consciousness.

Proceedings of the National Academy of Sciences of the United States of America, 101(17), 6809-6814.

Jacobsen, T., Horenkamp, T., \& Schröger, E. (2003). Preattentive memory-based comparison of sound intensity. Audiology \& Neuro-Otology, 8(6), 338-346.

Jacobsen, T., \& Schröger, E. (2001). Is there pre-attentive memory-based comparison of pitch? Psychophysiology, 38(4), 723-727. 
Jacobsen, T., \& Schröger, E. (2003). Measuring duration mismatch negativity. Clinical Neurophysiology : Official Journal of the International Federation of Clinical

Neurophysiology, 114(6), 1133-1143.

Jacobsen, T., Schröger, E., Horenkamp, T., \& Winkler, I. (2003). Mismatch negativity to pitch change: Varied stimulus proportions in controlling effects of neural refractoriness on human auditory event-related brain potentials. Neuroscience Letters, 344(2), 79-82.

Javitt, D. C. (2000). Intracortical mechanisms of mismatch negativity dysfunction in schizophrenia. Audiology \& Neuro-Otology, 5(3-4), 207-215.

Javitt, D. C., Grochowski, S., Shelley, A. M., \& Ritter, W. (1998). Impaired mismatch negativity $(\mathrm{MMN})$ generation in schizophrenia as a function of stimulus deviance, probability, and interstimulus/interdeviant interval. Electroencephalography and Clinical Neurophysiology, 108(2), 143-153.

Lijffijt, M., Lane, S. D., Meier, S. L., Boutros, N. N., Burroughs, S., Steinberg, J. L., et al. (2009). P50, N100, and P200 sensory gating: Relationships with behavioral inhibition, attention, and working memory. Psychophysiology, doi:10.1111/j.1469-8986.2009.00845.x. Lu, Z. L., Williamson, S. J., \& Kaufman, L. (1992). Human auditory primary and association cortex have differing lifetimes for activation traces. Brain Research, 572(1-2), 236-241. Malmierca, M. S., Cristaudo, S., Perez-Gonzalez, D., \& Covey, E. (2009). Stimulus-specific adaptation in the inferior colliculus of the anesthetized rat. The Journal of Neuroscience, 29(17), 5483-5493.

Mars, R. B., Debener, S., Gladwin, T. E., Harrison, L. M., Haggard, P., Rothwell, J. C., et al. (2008). Trial-by-trial fluctuations in the event-related electroencephalogram reflect dynamic changes in the degree of surprise. The Journal of Neuroscience, 28(47), 12539-12545.

Matuoka, T., Yabe, H., Ren, A., Hara, E., \& Kaneko, S. (2008). Memory trace dependence on number of stimuli in magnetic mismatch negativity. Neuroreport, 19(10), 1003-1007. 25Multiple time-scales of adaptation in the human auditory system May, P., Tiitinen, H., Ilmoniemi, R. J., Nyman, G., Taylor, J. G., \& Näätänen, R. (1999). Frequency change detection in human auditory cortex. Journal of Computational Neuroscience, 6(2), 99-120.

Näätänen, R. (1992). Attention and brain function. Hillsdale N.J. etc.: Lawrence Erlbaum Associates.

Näätänen, R., Gaillard, A. W., \& Mantysalo, S. (1978). Early selective-attention effect on evoked potential reinterpreted. Acta Psychologica, 42(4), 313-329.

Näätänen, R., Sams, M., Alho, K., Paavilainen, P., Reinikainen, K., \& Sokolov, E. N. (1988). Frequency and location specificity of the human vertex N1 wave. Electroencephalography and Clinical Neurophysiology, 69(6), 523-531.

Näätänen, R., Tervaniemi, M., Sussman, E., Paavilainen, P., \& Winkler, I. (2001). "Primitive intelligence" in the auditory cortex. Trends in Neurosciences, 24(5), 283-288.

Näätänen, R., Paavilainen, P., Rinne, T., \& Alho, K. (2007). The mismatch negativity (MMN) in basic research of central auditory processing: A review. Clinical Neurophysiology, 118(12), 2544-2590.

Näätänen, R., \& Winkler, I. (1999). The concept of auditory stimulus representation in cognitive neuroscience. Psychological Bulletin, 125(6), 826-859.

Nelken, I., \& Bar-Yosef, O. (2008). Neurons and objects: The case of auditory cortex. Frontiers in Neuroscience, 2(1), 107-113.

26Multiple time-scales of adaptation in the human auditory system

Nelken, I., Fishbach, A., Las, L., Ulanovsky, N., \& Farkas, D. (2003). Primary auditory cortex of cats: Feature detection or something else? Biological Cybernetics, 89(5), 397-406.

Perez-Gonzalez, D., Malmierca, M. S., \& Covey, E. (2005). Novelty detector neurons in the 
mammalian auditory midbrain. The European Journal of Neuroscience, 22(11), 2879-2885.

Picton, T. W., Woods, D. L., \& Proulx, G. B. (1978). Human auditory sustained potentials. II. stimulus relationships. Electroencephalography and Clinical Neurophysiology, 45(2), 198210.

Picton, T. W., Alain, C., Otten, L., Ritter, W., \& Achim, A. (2000). Mismatch negativity:

Different water in the same river. Audiology \& Neuro-Otology, 5(3-4), 111-139.

Reches, A., \& Gutfreund, Y. (2008). Stimulus-specific adaptations in the gaze control system of the barn owl. The Journal of Neuroscience, 28(6), 1523-1533.

Ritter, W., Sussman, E., Molholm, S., \& Foxe, J. J. (2002). Memory reactivation or reinstatement and the mismatch negativity. Psychophysiology, 39(2), 158-165.

Rosburg, T., Trautner, P., Korzyukov, O. A., Boutros, N. N., Schaller, C., Elger, C. E., et al. (2004). Short-term habituation of the intracranially recorded auditory evoked potentials P50 and N100. Neuroscience Letters, 372(3), 245-249.

Sams, M., Alho, K., \& Näätänen, R. (1983). Sequential effects on the ERP in discriminating two stimuli. Biological Psychology, 17(1), 41-58.

Sams, M., Alho, K., \& Näätänen, R. (1984). Short-term habituation and dishabituation of the mismatch negativity of the ERP. Psychophysiology, 21(4), 434-441.

Schröger, E., \& Wolff, C. (1996). Mismatch response of the human brain to changes in sound location. Neuroreport, 7(18), 3005-3008.

Squires, K. C., Wickens, C., Squires, N. K., \& Donchin, E. (1976). The effect of stimulus sequence on the waveform of the cortical event-related potential. Science (New York, N.Y.), 193(4258), 1142-1146.

Sussman, E., \& Winkler, I. (2001). Dynamic sensory updating in the auditory system. Brain Research.Cognitive Brain Research, 12(3), 431-439.

Ulanovsky, N., Las, L., \& Nelken, I. (2003). Processing of low-probability sounds by cortical neurons. Nature Neuroscience, 6(4), 391-398.

Ulanovsky, N., Las, L., Farkas, D., \& Nelken, I. (2004). Multiple time scales of adaptation in auditory cortex neurons. The Journal of Neuroscience, 24(46), 10440-10453.

Winkler, I., Karmos, G., \& Näätänen, R. (1996). Adaptive modeling of the unattended acoustic environment reflected in the mismatch negativity event-related potential. Brain Research, 742(1-2), 239-252.

Winkler, I. (2007). Interpreting the mismatch negativity. Journal of Psychophysiology, 21(3), 147-163. 